\title{
Results from the super Rosenbluth separation experiment
}

Ralph Segel, for the JLab E01-001 collaboration

Northwestern University, Evanston, Illinois 60208, USA

Received: 8 October 2004 / Published Online: 8 February 2005

(C) Società Italiana di Fisica / Springer-Verlag 2005

\begin{abstract}
We present preliminary results from Rosenbluth separations for elastic $p(e, p)$ scattering performed at $Q^{2}=2.64,3.20$, and $4.10 \mathrm{GeV}^{2}$. Several improvements were made in the technique leading considerably higher precision than achieved in previous measurements. The electric $\left(G_{E}\right)$ and magnetic $\left(G_{M}\right)$ form factors were found to approximately "scale", i.e. $\mu_{p} G_{E} / G_{M} \approx 1$, consistent with previous Rosenbluth separation results but inconsistent with polarization transfer measurements which report $\mu_{p} G_{E} / G_{M}$ falling linearly with $Q^{2}$.
\end{abstract}

PACS. 25.30.Bf Elastic electron scattering - 13.40.Gp Electromagnetic form factors - 14.20.Dh Proton and neutron properties

The Rosenbluth formula [1] expresses the cross section for $e-p$ elastic scattering in terms of the proton's electric $\left(G_{E}\right)$ and magnetic $\left(G_{M}\right)$ form factors:

$$
\frac{d \sigma}{d \Omega_{e}}=\frac{\sigma_{M o t t}}{\varepsilon(1+\tau)}\left(\tau G_{M}^{2}+\varepsilon G_{E}^{2}\right),
$$

where $\tau=Q^{2} / 4 M_{p}^{2}$, $\varepsilon$ is the longitudinal polarization of the exchanged virtual photon, $\varepsilon=\left(1+2(1+\tau) \tan ^{2}\left(\theta_{e} / 2\right)\right)^{-1}$, $M_{p}$ is the proton mass and $\theta_{e}$ the electron scattering angle. The form factors depend only on momentum transfer and so Rosenbluth separations are performed by varying the incident electron energy and the scattering angle so as to leave $Q^{2}$ constant and by varying $\varepsilon$ extract $G_{E}$ and $G_{M}$. Such measurements have been reported from 1970 to the present day 2,3 . Both form factors were found to follow the dipole form (Fig. 11) with the ratio $\mu_{p} G_{E} / G_{M}$ appearing to cluster about its asymptotic value of unity where $\mu_{p}$ is the proton magnetic moment in units of the

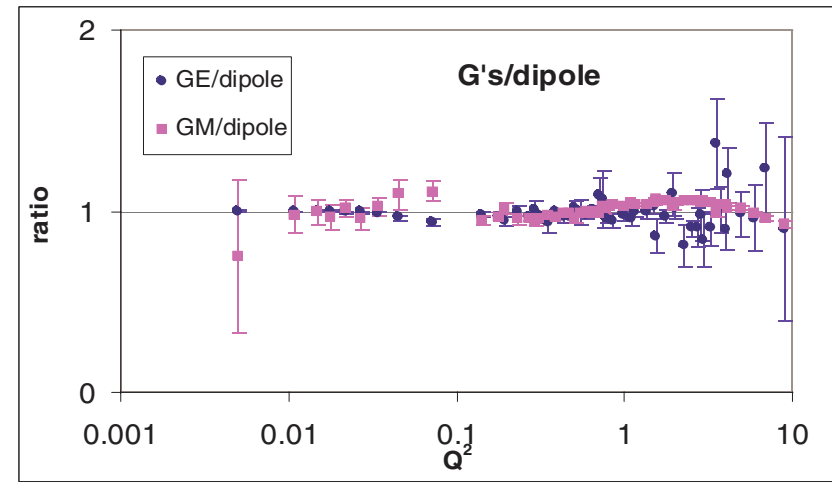

Fig. 1. Values of $G_{E} / G_{D}$ and $G_{M} /\left(\mu_{p} G_{D}\right)$ obtained in previous Rosenbluth separation experiments [2], where $G_{D}$ is the dipole form, $G_{D}=1 /\left(1+Q^{2} / 0.71\right)^{2}$

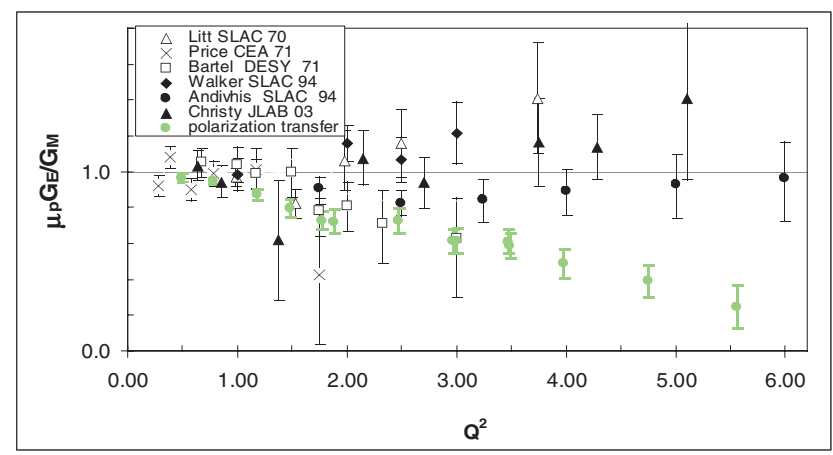

Fig. 2. The ratio of electric to magnetic form factor as determined in previous Rosenbluth separation 23.3 (black) and polarization transfer [6, 8, (green) experiments

Bohr magneton. This scaling behavior of $\mu_{p} G_{E} \approx G_{M}$ is supported by fits to the data [2,4] and came to be generally accepted.

An alternate method for measuring the proton form factors was proposed by Arnold, Carlson and Gross [5] in which the incident electron beam is longitudinally polarized in which case the recoil proton is both longitudinally and transversely polarized and the ratio of form factors is proportional to the ratio of polarizations, i.e.:

$$
\frac{G_{E}}{G_{M}}=-\frac{P_{t}}{P_{l}} \frac{E_{e}+E_{e}^{\prime}}{2 M_{p}} \tan \left(\theta_{e} / 2\right),
$$

where $P_{t}$ and $P_{l}$ are the transverse and longitudinal polarizations, respectively, of the recoil proton. This ratio has been measured [6,7,8] with the result that $G_{E} / G_{M}$ falls off linearly with increasing $Q^{2}$ and does not scale. Results from the two techniques are shown in Fig. 2. The uncertainties on the Rosenbluth derived ratios are much larger and show a large scatter which led to speculation that at 


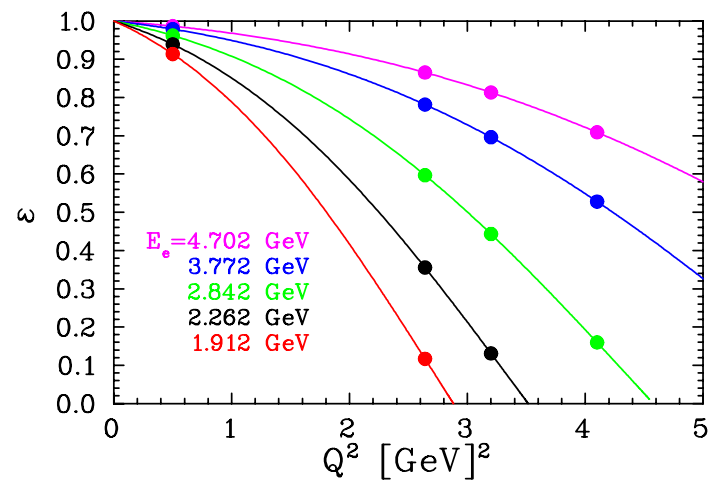

Fig. 3. Kinematic points at which proton spectra were measured

$Q^{2} \gtrsim 2 \mathrm{GeV}^{2}$, where the electric contribution is but a small fraction of the total cross section, the Rosenbluth determinations of $G_{E}$ were unreliable. The goal of the $\mathrm{Su}-$ per Rosenbluth experiment was to measure $G_{E} / G_{M}$ in the $Q^{2}=2-5 \mathrm{GeV}^{2}$ range with uncertainties comparable to those obtained in the polarization transfer experiments. This goal was achieved.

The experiment was performed in Hall A of Jefferson Lab using a liquid hydrogen target and the HRS spectrometers to detect the recoil protons. This is in contrast to all previous Rosenbluth separations where the electrons were detected. Detecting the protons has several very important advantages:

1. The proton momentum is constant at constant $Q^{2}$ thus eliminating the effect of any momentum dependent detection efficiencies.

2 . The proton cross section $\left(d \sigma / d \Omega_{p}\right)$ changes by less than a factor of 2 over the $\varepsilon$ range covered here while the electron cross section $\left(d \sigma / d \Omega_{e}\right)$ varies by almost 2 orders of magnitude. Furthermore, the minimum cross section is twenty times larger for the proton. Thus by detecting the proton count rate effects on the extracted form factor ratio were negligibly small and yet it was possible to obtain high statistics spectra at all points.

3. At the most sensitive points the sensitivity of the proton cross section to angle is half the sensitivity of the electron cross section.

4. Radiative corrections are more than a factor of 2 smaller and, most importantly, much less $\varepsilon$ dependent.

Rosenbluth separations were performed at $Q^{2}=2.64$, 3.20 and $4.10 \mathrm{GeV}^{2}$. The kinematics of the experiment are shown in Fig. 3. Figure 4 shows the spectra of "missing momentum" $\left(\delta_{p}\right)$, the difference between the measured proton momentum and that expected at the measured proton angle. The elastic peak clearly dominates the spectra. The width of the peak is largely due to the angular resolution of the tracking system and is well fit by the Monte Carlo simulation. There is a background that extends over the entire $\delta_{p}$ region due to reactions, primarily quasielastic scattering, in the target endcaps. This background could be accurately determined by taking data at each point with a dummy target. The other significant background is protons from the $\gamma \mathrm{p} \rightarrow \pi^{0} \mathrm{p}$ reaction initiated by bremsstrahlung. This background, which cuts off
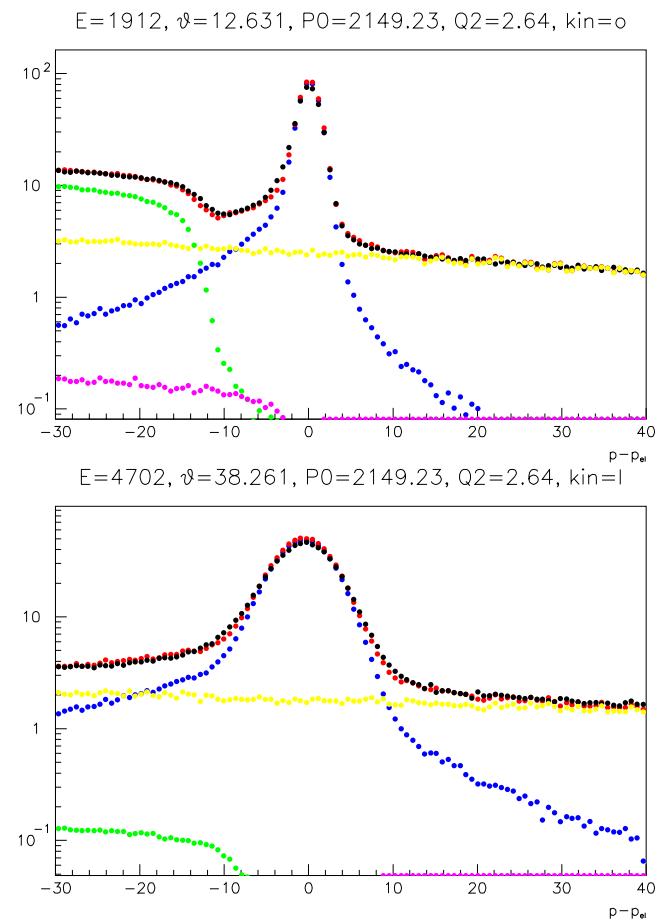

Fig. 4. Spectra at $Q^{2}=2.64 \mathrm{GeV}^{2}$ at a small $\varepsilon$, small $\theta_{p}$ and a large $\varepsilon$, large $\theta_{p}$ point. Shown are the decomposition of the experimental spectra (black) into the simulated $p(e, p)$ elastic peak (blue), reactions in the endcaps (yellow), simulated $\gamma p \rightarrow \pi^{0} p$ (green) and simulated $\gamma p \rightarrow \gamma p$ (purple)

below the elastic peak because of the finite pion mass, can be accurately simulated by folding an $\mathrm{s}^{-7}$ cross section dependence into a calculated bremsstrahlung spectrum.

The simulation used the Monte Carlo code SIMC and took into account the acceptance, angular and momentum resolution of the spectrometer, scattering and absorption of the protons, and incorporated radiative corrections based upon the work of Mo and Tsai 9, 10. Coincidence $\left(e, e^{\prime} p\right)$ spectra were taken and used to check the shape of the calculated elastic peak. Cross sections were determined by subtracting off the backgrounds and then determining the factor needed to normalize the experimental elastic peak to that calculated by the Monte Carlo.

While one spectrometer was analyzing the protons of interest the other was set at $Q^{2}=0.5 \mathrm{GeV}^{2}$. Here the elastic cross sections are very large and the difference between the polarization transfer and the Rosenbluth form factors small. Furthermore, while a large range of $\varepsilon$ was being covered at the high $Q^{2}$ only a very small range $(<0.1)$ was covered at $0.5 \mathrm{GeV}^{2}$ (Fig. 3) and thus the second HRS acted as a luminosity monitor.

Absolute cross sections were determined to about $3 \%$ and relative cross sections at the same $Q^{2}$ to about $0.8 \%$. Figure 5 shows reduced cross sections:

$$
\sigma_{r}=1+(\varepsilon / \tau)\left(G_{E} / G_{M}\right)^{2}
$$

at $2.64 \mathrm{GeV}^{2}$ plotted as a function of $\varepsilon$. The slope is close to that expected for scaling and not consistent with that predicted by the polarization transfer experiments. Similar results were obtained at the other two $Q^{2}$ points. Fig- 


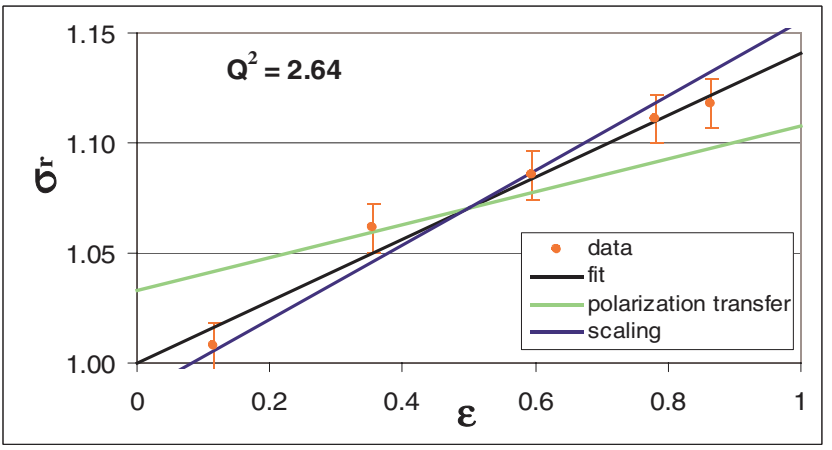

Fig. 5. Reduced cross section (see text) as a function of $\varepsilon$. A linear fit to the data is shown as are the predictions of scaling and of the polarization transfer experiments

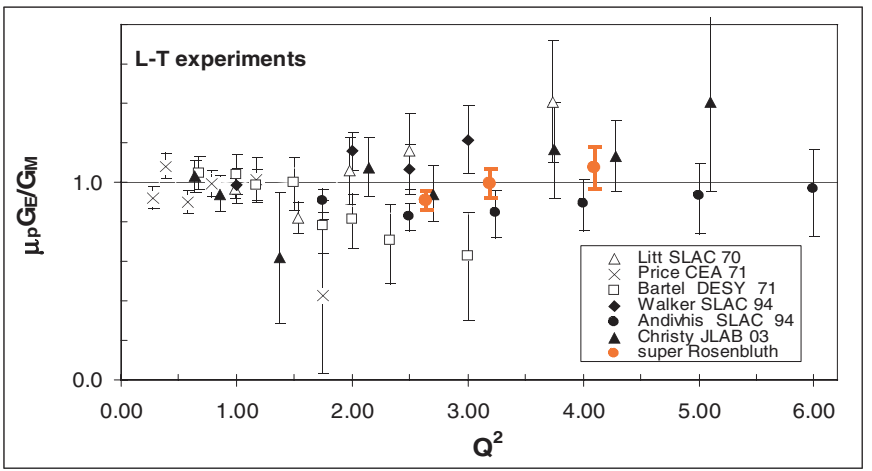

Fig. 6. $G_{E} / G_{M}$ from all reported Rosenbluth separation experiments including the present work

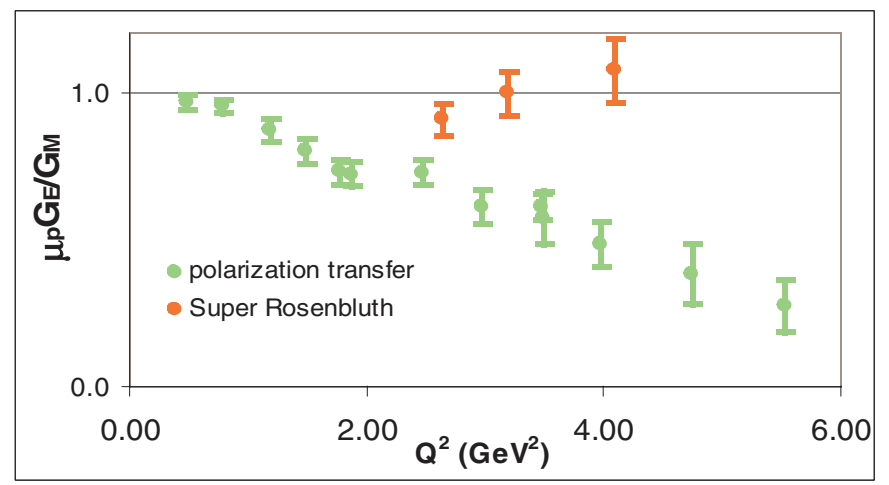

Fig. 7. The present results compared to the results of the polarization transfer experiments [6], 8$]$. Also shown is a fit to all previous Rosenbluth data [1]

ure 6 shows all of the reported $e-p$ elastic Rosenbluth separation values for $G_{E} / G_{M}$ including those from the present work. All of the experiments are basically consistent, with the uncertainties in the present experiment the smallest by far. The fact that the present experiment where protons were detected agrees with the experiments where the electrons were detected demonstrates that the radiative corrections are well understood. The present results are compared to the polarization transfer results in Fig. 7 They do not agree!

While there have been many Rosenbluth experiments done at different labs by different groups the polariza- tion transfer experiments in this $Q^{2}$ range have all been done by the same group using the same apparatus. An experiment to measure the asymmetry in the scattering of longitudinally polarized electrons by polarized protons, which would provide an independent check, has been proposed [12] and hopefully will be performed soon. In addition, there is an approved experiment for Hall $\mathrm{C}$ at Jefferson Lab that will extend polarization transfer to larger $Q^{2}$ values using a different spectrometer and polarimeter from the existing measurements $[13$.

Two-photon exchange is not fully treated in the Mo and Tsai prescriptions and estimates [14 15, 16, 17] of the effect of these neglected terms indicate that they could change the cross sections enough to lower $G_{E}$ down to close to what the polarization transfer experiments imply while the effect on the polarization transfer is much smaller. There are several other possible manifestations of two-photon exchange terms, one of which is a non-linearity in the $\varepsilon$ dependence of the reduced cross sections. An experiment to extend the techniques of the super Rosenbluth experiment to search for such a non-linearity has been proposed [18. Another manifestation of two-photon exchange effects would be an $\varepsilon$ dependence of the polarization transfer and an experiment has been approved to measure the polarization transfer over a wide range of $\varepsilon$ [19]. Until it has been demonstrated that the difference between the Rosenbluth and the polarization transfer $G_{E} / G_{M}$ is due to heretofore neglected two-photon exchange effect the discrepancy must be regarded as an open issue that must be resolved.

\section{References}

1. M.N. Rosenbluth, Phys. Rev. 79 , 615 (1950)

2. R.C. Walker et al.: Phys. Rev. D 49, 5671 (1994)

3. M.E. Christy et al.: Phys. Rev. C 70, 015206 (2004)

4. J. Arrington: Phys. Rev. C 68, 034325 (2003)

5. R.G. Arnold, C.E. Carlson, F. Gross: Phys. Rev. C 23, 363 (1981)

6. M.K. Jones et al.: Phys. Rev. Lett. 84, 1398 (2000)

7. O. Gayou et al.: Phys. Rev. C 64, 038202 (2001)

8. O. Gayou et al.: Phys. Rev. Lett. 88, 092301 (2002)

9. L.W. Mo, Y.-S. Tsai: Rev. Mod. Phys. 41, 205 (1969)

10. R. Ent et al.: Phys. Rev. C 64, 054610 (2001)

11. J. Arrington: Phys. Rev. C 69, 022201 (2004)

12. X. Zheng, J.R. Calarco, O.A. Rondan, spokespersons: JLab proposal E04-111

13. E. Brash, C. Perdrisat, V. Punjabi, spokespersons: JLab experiment E01-109

14. P.A.M. Guichon, M. Vanderhaeghen: Phys. Rev. Lett. 91, 142303 (2003)

15. P.G. Blunden, W. Melnitchouk, J.A. Tjon: Phys. Rev. Lett. 91, 142304 (2003)

16. Y.C. Chen, A. Afanasev, S.J. Brodsky, C.E. Carlson, M. Vanderhaeghen: arXiv:hep-ph/0403058

17. J. Arrington: arXiv:hep-ph/0408261 (2004)

18. J. Arrington, spokesperson: JLab proposal E04-106

19. R. Gilman, L. Pentchev, C. Perdrisat, R. Suleiman, spokespersons: JLab experiment E01-019 